\title{
Fundamentals of Optical Imaging
}

\author{
Ralf B. Schulz(区) and Wolfhard Semmler
}

1 Introduction and Overview $\ldots \ldots \ldots \ldots \ldots \ldots \ldots \ldots \ldots \ldots \ldots \ldots \ldots \ldots \ldots \ldots \ldots \ldots$

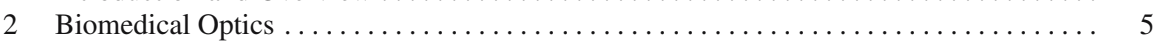

2.1 Photon Propagation in Tissues $\ldots \ldots \ldots \ldots \ldots \ldots \ldots \ldots \ldots \ldots \ldots \ldots \ldots \ldots \ldots$

2.2 Bioluminescence and Fluorescence $\ldots \ldots \ldots \ldots \ldots \ldots \ldots \ldots \ldots \ldots \ldots \ldots$

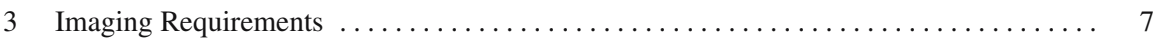

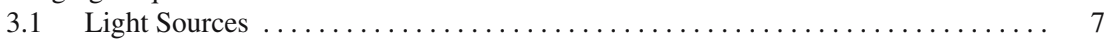

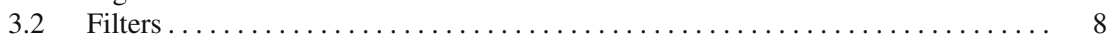

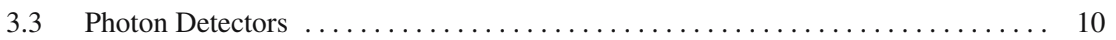

4 Microscopic Imaging Techniques $\ldots \ldots \ldots \ldots \ldots \ldots \ldots \ldots \ldots \ldots \ldots \ldots \ldots \ldots \ldots$

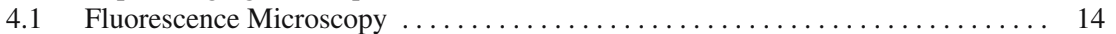

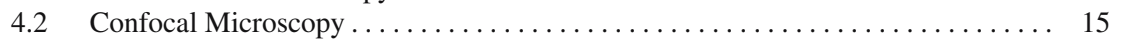

$4.34 \pi$ Microscopy $\ldots \ldots \ldots \ldots \ldots \ldots \ldots \ldots \ldots \ldots \ldots \ldots \ldots \ldots \ldots \ldots$

4.4 Stimulated Emission Depletion (STED) $\ldots \ldots \ldots \ldots \ldots \ldots \ldots \ldots \ldots \ldots \ldots \ldots$

4.5 Other Microscopic Techniques $\ldots \ldots \ldots \ldots \ldots \ldots \ldots \ldots \ldots \ldots \ldots \ldots \ldots \ldots \ldots$

5 Whole-Animal Imaging Techniques $\ldots \ldots \ldots \ldots \ldots \ldots \ldots \ldots \ldots \ldots \ldots \ldots \ldots \ldots \ldots \ldots$

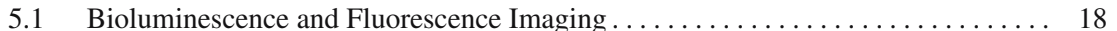

5.2 Bioluminescence and Fluorescence Tomography . . . . . . . . . . . . . 19

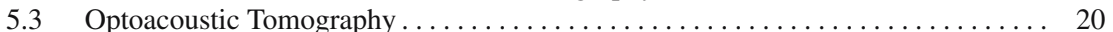

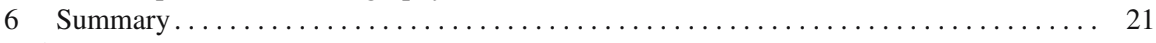

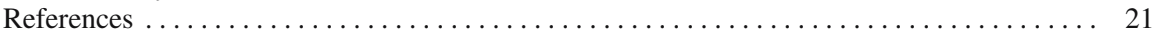

Abstract Optical imaging techniques offer simplistic while highly sensitive modalities for molecular imaging research. In this chapter, the major instrumental necessities for microscopic and whole-animal imaging techniques are introduced. Subsequently, the resulting imaging modalities using visible or near-infrared light are presented and discussed. The aim is to show the current capabilities and application fields of optics.

Ralf B. Schulz

Helmholtz Zentrum München Institut für Biologische und Medizinische Bildgebung, Ingolstädter Landstraße 1, 85764 Neuherberg, Germany

ralf.schulz@helmholtz-muenchen.de

W. Semmler and M. Schwaiger (eds.), Molecular Imaging I.

Handbook of Experimental Pharmacology 185/I.

(C) Springer-Verlag Berlin Heidelberg 2008 


\section{Introduction and Overview}

Imaging techniques employing visible light have been a standard research tool for centuries: vision is usually our most developed sense, and thus the visual inspection of a specimen has always been a scientist's first choice. The development of lenses, telescopes, and microscopes has helped us visually explore large or small worlds previously not accessible. In biomedical research, with the discovery of fluorescence, fluorescence microscopy has become the technique of choice for single-cell imaging (Vo Dinh 2003a). Novel scanning techniques, as described further below, yield high resolution by overcoming the diffraction limit usually connected to lensbased systems. Thus, they allow tomographic imaging of individual cells without slicing them, as necessary for electron microscopy. This has led to a number of discoveries, including the tubular structure of mitochondria (Hell 2003).

The capabilities of fluorescence microscopes have in turn sparked further technological advances in fluorescent markers and probe systems. The discoveries of bioluminescent and fluorescent proteins have enabled biologists to produce cells that synthesize optically active markers by themselves, a fundamental simplification for gene expression imaging (Massoud and Gambhir 2003).

Compared with other types of contrast agents, optical probes offer unique imaging capabilities: not only can they be targeted to receptors, like radioactive tracers or MR-active substances, but fluorescent probes can also be activated due to enzymatic reactions (activatable probes), and they can be produced by cells themselves in the form of bioluminescent enzymes or fluorescent proteins (Hoffman 2005). Fluorescent proteins nowadays can be engineered to emit in the far red, necessary for in vivo applications (Shaner et al. 2005).

However, these advantages come with several caveats: compared with radiotracers, fluorescent molecules are big, relatively unstable (they are affected by photobleaching), and some of them are cytotoxic to some degree. Furthermore, biological tissues are highly diffuse; visible light is scattered within a few microns. Fluorescence-based imaging techniques are thus often either not applicable in vivo, only applicable to very superficial regions due to the scattering, nonquantitative, or highly experimental and not yet available for daily routine, as is the case for optical tomographic applications.

Over the past few years, optical imaging technologies for whole-animal imaging (or even patient-based imaging) have attracted more and more attention, the reason being that an abundance of highly specific optical probes is nowadays available for in vitro applications that would be of much help if applied in vivo (Weissleder and Ntziachristos 2003). Optical imaging is hoped to provide a reliable way of translating in vitro research to in vivo. Most of these techniques are planar [two-dimensional (2D)]. The advances in computer technology and mathematical modeling have also led to the development of optical tomographic (3D) techniques. For a current review of available techniques, please refer to Gibson et al. (2005) or Hielscher (2005).

This chapter will first introduce the basics of optical imaging and provide recommendations for further reading. While a couple of years ago, it was hard to find comprehensive summaries of optical imaging techniques, a number of complete books 
and reviews have now been published by various groups and are recommended to the interested reader, such as the books edited by Vo Dinh (2003a), Mycek and Pogue (2003), and Kraayenhof et al. (2002). Due to length constraints, this article can only concentrate on a few key points.

\section{Biomedical Optics}

\subsection{Photon Propagation in Tissues}

The fundamental limits for optical imaging in terms of either penetration depths or resolution are given by the optical properties of tissue; due to the many very small structures and boundaries in cells, tissue becomes highly scattering and absorbing for photons in the visible range. Absorption and scattering are measured in terms of the absorption coefficient, $\mu_{a}$, and the scattering coefficient, $\mu_{s}$, with physical units of $\mathrm{cm}^{-1}$ or $\mathrm{mm}^{-1}$. The reciprocal value of these coefficients yields the mean free path.

While tissue is strongly absorbing for light having a short wavelength $\left(\mu_{a} \gg\right.$ $1 \mathrm{~cm}^{-1}$, resulting in a mean free path of much less than $1 \mathrm{~cm}$ ) caused by the most common absorbers in cells, cytochromes and hemoglobin, light in the near infrared range (NIR) between 600 and $900 \mathrm{~nm}$ can penetrate several centimeters deep into tissue $\left(\mu_{a}<0.5 \mathrm{~cm}^{-1}\right.$, even down to $\mu_{a} \approx 0.1 \mathrm{~cm}^{-1}$; Fig. 1 , yielding a mean free path of up to $10 \mathrm{~cm}$ if only absorption is taken into account). This wavelength region

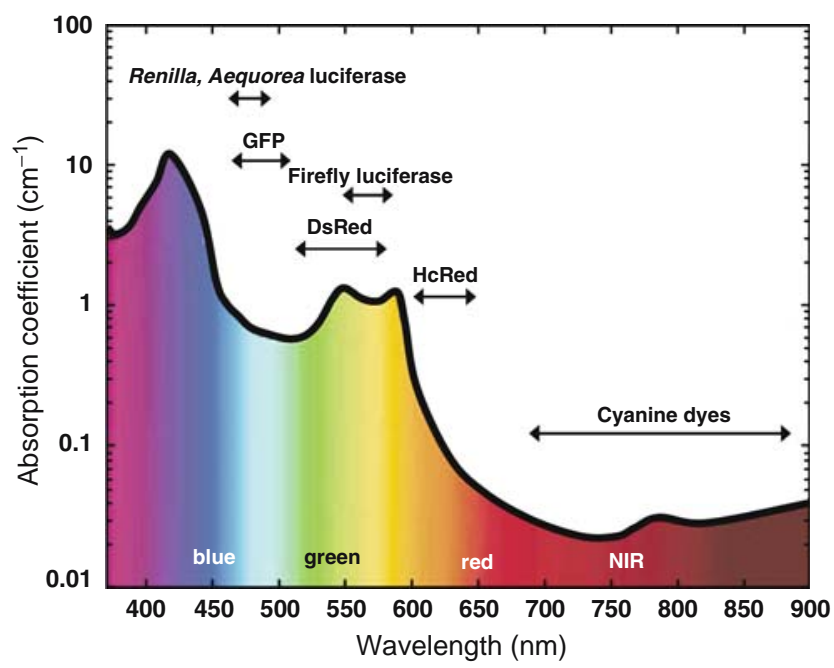

Fig. 1 Absorption and autofluorescence of tissue, depending on wavelength. The wavelength regions of important dyes are indicated by arrows. (Adapted from Weissleder and Ntziachristos 2003) 
has been termed the "water window," as for longer wavelengths water absorption becomes the dominant term (Weissleder and Ntziachristos 2003).

The low absorption in the NIR wavelength range has led to the development of an abundance of NIR fluorescent molecules. However, in general, these fluorochromes are less efficient and less bright than their short-wavelength counterparts. This also implies that for a certain application the wavelength has to be chosen very carefully: in a more absorbing wavelength range, the increase in efficiency and stability of the molecules might outweigh the disadvantages of higher absorption.

The main problem when using visible photons, however, is not attenuation but scattering, with a scattering coefficient in the order of $\mu_{s} \approx 100 \mathrm{~cm}^{-1}$, being about four orders of magnitude larger than absorption and thus leading to a total mean free path of only $0.1 \mathrm{~mm}$. Scattering results from the many different diffracting interfaces present in the cells of which tissue is comprised. Scattering due to cells is largely anisotropic, with an average scatter angle of only $25^{\circ}$.

Standard methods for scatter reduction, as known for example from nuclear imaging, will fail in these cases due to the extreme number of scattering events that detected photons have undergone. Scattering decreases with longer wavelengths, but otherwise it remains relatively constant over the visible range (contrary to the sharply peaked absorption spectra of biological chromophores, Fig. 1).

When choosing an appropriate fluorochrome or the optimal wavelength for a specific imaging purpose there is also another, counter-intuitive effect one might have to take into account: the choice of a wavelength in a strongly absorbing region will result in the preferential detection of photons that have undergone fewer scattering events, as scattering increases the length of the propagation path, and the higher absorption will constrain path lengths. Thus, scattering can be significantly reduced; however, signal intensities are decreased as well.

For a comprehensive list of tissue optical properties and according references, please refer to the review by Mobley and Vo-Dinh (2003).

\subsection{Bioluminescence and Fluorescence}

The term fluorescence refers to the emission of a photon caused by a molecule's transition from an excited electronic state to (usually) its ground state. Both states have the same spin multiplicity, which makes fluorescence a singlet-singlet transition. Fluorescent molecules often consist of a more or less a long chain of carbon atoms between two aromatic structures, which as a whole acts as an optical resonator. The length of the chain is related to the emission wavelength.

The excited state is reached by absorption of a photon with sufficient energy, i.e., of a photon of higher energy (shorter wavelength) than the energy difference between excited and ground state. The wavelength difference between the wavelength of maximum absorption and the emission wavelength is called Stokes shift. A large Stokes shift facilitates the creating of filters blocking the excitation light. Shifts range between less than $20 \mathrm{~nm}$ and several hundred nanometers. The lifetime of the excited state is termed the fluorescence lifetime, $\tau[\mathrm{s}]$, and usually amounts to 
a time span between some $100 \mathrm{ps}$ to several nanoseconds. For singlet-triplet transitions (phosphorescence) occurring for example in some lanthanides, even lifetimes of several milliseconds can be observed.

The probability that the transition from excited to ground state will occur by emission of a photon is called the quantum yield, $\gamma$, and is a measure for fluorochrome efficiency. The absorption efficiency is described by the molar extinction coefficient, $\varepsilon\left[\mathrm{mol}^{-1} \mathrm{~cm}^{-1}\right]$. The total absorption created by the fluorochrome can be calculated using the relation $\mu_{a}=\varepsilon c$, where $c$ [mol/l] is the fluorochrome concentration. It is important to notice that all these factors, including the emission and absorption spectra, are influenced by the chemical environment ( $\mathrm{pH}$ value, etc). Please refer to Redmond (2003) or Lakowicz (2006) for details.

Bioluminescence is a special form of chemoluminescence. Photons are emitted when a bioluminescent enzyme (e.g., luciferase) metabolizes its specific substrate (e.g., luciferin). As in this case no excitation light is necessary to produce a signal, there is also no background, i.e., neither from autofluorescence nor from filter leakage. However, while it is relatively easy to guide light to fluorescent probes, it is hard to ensure that the substrate is transported to all possibly bioluminescent cells (Massoud and Gambhir 2003).

\section{Imaging Requirements}

Optical imaging of any kind requires three fundamental system parts: light sources to induce the desired signal, filters to eliminate background signal, and photon detectors to acquire the signal. These components will be discussed in the following. For a detailed overview please refer to Vo-Dinh (2003b) or Lakowicz (2006).

\subsection{Light Sources}

Light sources can generally be distinguished by their emission spectra, emission power, as well as their capabilities concerning pulsing or modulation. Fluorescence excitation is usually performed with one of the following:

- High-pressure Arc Lamps: They exhibit strong, nearly continuous emission between $200 \mathrm{~nm}$ (UV) and 1,000 nm (IR). These high intensity sources are of major interest in cell biology and spectroscopy but are less often used in whole animal imaging.

- Light-emitting diodes: Due to the recent developments of extremely luminous LEDs ("LumiLEDs") and the availability of all kinds of emission spectra between $300 \mathrm{~nm}$ and $700 \mathrm{~nm}$, they have become a very cheap and stable alternative to lasers, if coherence is unimportant or even undesirable, e.g., due to speckle noise. To sharpen their emission spectrum, one usually combines LEDs with a filter system (Fig. 2). 
a

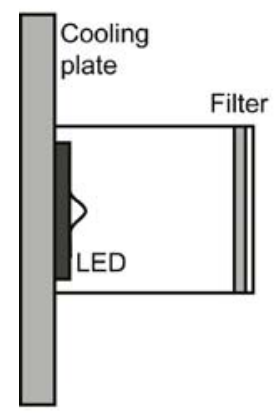

b

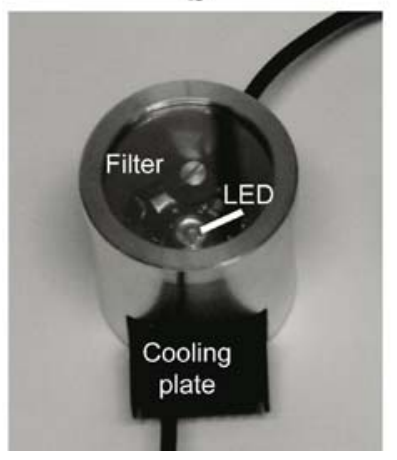

C

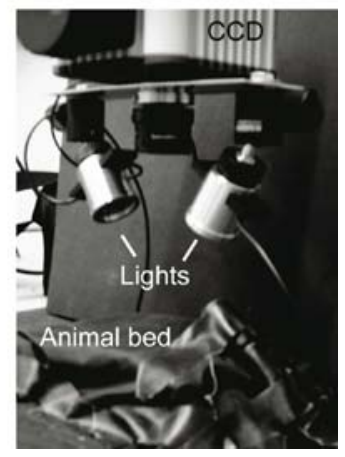

Fig. 2 a, b An LED light source for in vivo imaging. Light from a luminous LED, emitting isotropically in all directions, is filtered and can excite superficially located fluorochromes in mice. a Schematic drawing of the light source. b Close-up image of the source. c Application example

- Lasers: A standard tool in biomedical imaging. They are available in the form of solid state lasers (diodes), especially in the near infrared, red or green, but nowadays even blue; as gas lasers; as tunable dye lasers (usually pumped by gas lasers); or as nonlinear lasers that produce also IR output. The output of laser diodes can be continuous, pulsed, or modulated with frequencies of up to $100 \mathrm{MHz}$. Lasers exhibit a sharply peaked (monochromatic) spectrum, coherent and usually polarized light output. There are lasers available that can be tuned to different wavelengths.

\subsection{Filters}

Crucial for the signal-to-noise ratio of optical systems is the performance of the filters used. While optical signals are usually weak, one of the biggest issues is elimination of excitation light, as its wavelength is usually close to the emission, and at the same time, the signal is much stronger due to the limited quantum yield and the limited solid angle observable.

Filters are distinguished by their transmission spectra. Neutral density filters absorb a constant fraction of light, independent of wavelength. They are characterized by their optical density $(\mathrm{OD})$, defined as $\mathrm{OD}=\log _{10}\left(\mathrm{I}_{\mathrm{t}} / \mathrm{I}_{0}\right)$, with $\left(\mathrm{I}_{\mathrm{t}} / \mathrm{I}_{0}\right)$ being the ratio of transmitted to original light intensity. Longpass and shortpass filters transmit light above and below a certain wavelength, respectively. Bandpass filters transmit a (narrow) light-band, characterized by its central wavelength and the full width half maximum of the transmission band. See Fig. 3 for examples of bandpass and longpass transmission spectra. Notch filters (sometimes called band-rejection filters) suppress the transmission of a narrow light band. They are characterized analogously to bandpass filters by central blocked wavelength, and the FWHM of the blocked band. 


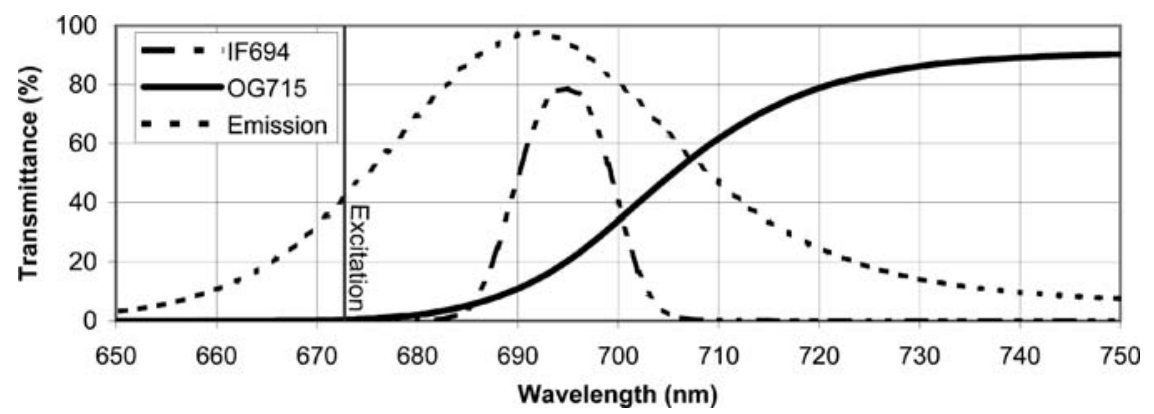

Fig. 3 Two possible filters for imaging of the Cy5.5 fluorochrome, a bandpass (IF694, LaserComponents, Germany) with peak transmission at $694 \mathrm{~nm}$, and a long pass filter (OG715, Schott, Germany). Although the glass filter blocks significantly more light at the peak wavelength of the fluorochrome $(695 \mathrm{~nm})$, due to the red tail of the emission spectrum, total transmission is $30 \%$, while for the interference filter, it is only $19 \%$. The emission spectrum is shown as a dotted line. The usual excitation wavelength of $672 \mathrm{~nm}$ is indicated by a vertical line

Most of the available filters to date are based either on absorption, interference, or dispersion. The differences are described in the following.

\subsubsection{Absorption-based Filters}

In absorbing filters, light is either transmitted through the filter or absorbed in it. The filter itself consists of the absorber and a substrate, which is commonly either a gel or glass. The advantage is their low cost and the independence of transmission properties of the angle of incidence, contrary to interference-based filters (see below). Disadvantages are the relatively low specificity, i.e., these filters usually exhibit smooth transmission curves, which make it difficult to filter in the case of small Stokes shifts. Furthermore, as blocked light is absorbed, these filters are only suitable for low intensities. Gel filters additionally are prone to bleaching, and sensitive to heat or humidity.

Due to their smooth transmission spectra, absorption filters are mostly used as neutral density filters, or long- and shortpass filters. Absorption-based bandpasses do exist, but they have large FWHMs and are usually not suitable for fluorescence detection.

\subsubsection{Interference-based Filters}

Interference filters either transmit or reflect light. Thus, nearly no energy is absorbed by the filter, which makes them suitable for filtering very intense light. They consist of a number of dielectric layers that partially reflect incoming light. The distance between these layers is chosen such that interference occurs in a way that transmitted light constructively interferes in the forward direction, while destructively 
interfering in the backwards direction; blocked light needs to destructively interfere in the forward direction and constructively interfere in the backward direction.

Thus, these filters can be manufactured to be much more specific than absorbing filters, as can be seen in Fig. 3, where the transmission curves of the bandpass are much steeper than these of the absorbing longpass. However, the interference effects strongly depend on the angle of incidence; it is shortest for light rays incident at an angle of $0^{\circ}$ (perpendicular to the surface), while the distances geometrically increase for larger angles. Thus, interference filters can only be used for parallel light as used in a fluorescence microscope (see below) but not in front of an objective.

\subsubsection{Dispersive Elements}

Dispersion is the wavelength-dependence of photon propagation speed in media. When light enters a dispersive medium, it is split into its spectral components as the diffraction angle will depend on wavelength. A typical example is an optical prism. Dispersive media can be used to filter out light if only parts of the resulting spectrum are used for illumination or detection, as in a photospectrometer. Typical examples of such dispersive media are diffraction gratings, which most often are comprised of a large number of grooves on a highly reflective surface. The distance between adjacent grooves determines the spectral properties of the grating.

Dispersive effects can also be used to create acoustically tunable bandpass filters (AOTFs) or liquid crystal tunable filters (LCTFs), novel types of devices that only recently found their way into biomedical imaging. In AOTFs, a standing acoustic wave is induced in a birefringent crystal to create spatially varying changes in refractive index via the acousto-optical effect. This wave pattern of changing refractive indices acts like a Bragg grating and thus can be used as a reflective monochromator. In LCTFs, a refractive index change is generated by alignment of the liquid crystal molecules in an externally applied electrical field.

The advantages of dispersive variable band-pass filters are their fast adjustment times (less than $100 \mathrm{~ms}$ ) and narrow filtering possibilities (up to $1 \mathrm{~nm}$ FWHM). Filtering requires, however, exactly parallel light with normal incidence on the filter.

\subsection{Photon Detectors}

Characteristic properties of photon detectors are the number of measurement channels they provide (single channel or multichannel devices), their dynamics (for digitized signals commonly expressed in bits), sensitivity (in terms of the quantum efficiency, i.e., the probability that a single photon creates a signal), and time resolution.

In this section we distinguish between photon counting detectors-analog detectors that produce individual signals for each incoming photon as used in extreme low-light or time-resolved applications - and integrating detectors without inherent 
time resolution. Intensified imaging devices, consisting of a combination of the previous two types, are considered a third category. They allow for time-resolved acquisition of large-field images due to their gating capabilities.

\subsubsection{Photon Counting Devices}

In photon counting devices, an incident photon is absorbed and subsequently generates some kind of charge displacement via the photoelectric effect, which is then amplified by several orders of magnitude to result in a measurable current. Using suitable read-out electronics, this signal can be detected in real-time. The amplification can also be used to employ these devices as image intensifiers; see 3.3.3.

Two imaging modes are commonly applied. When a pulsed light source is used, counted photons can be related to the time of the source pulse, and thus provide information about the optical path length of these photons. The path length in turn is indirectly related to the amount of scattering photons have undergone. Scattering elongates the propagation path compared with a straight, unscattered propagation path. In this way, scattering and absorbing inclusions in tissue can be distinguished. This mode of operation is termed time-domain detection. If instead of a pulsed light source, a modulated light source is employed, the detection technique is termed frequency-domain detection. Here, the phase shift between light-source modulation and detected signal is examined, from which it is, for instance, related to the lifetime of a fluorochrome and the optical properties of tissue.

Single-channel photon-counting devices are commonly photomultiplier tubes (PMTs) (Fig. 4a), as known from nuclear imaging, and (avalanche) photodiodes.

a

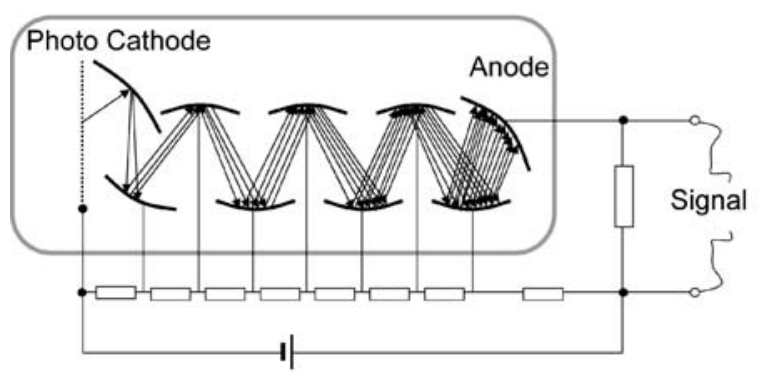

b

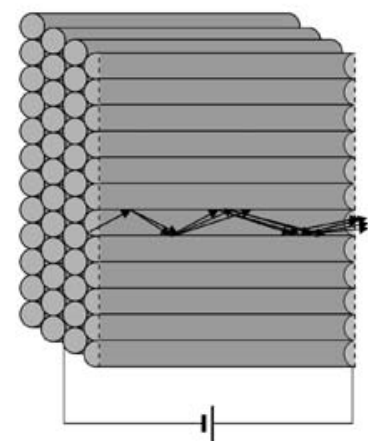

Fig. 4 a, b Light amplification via the photoelectric effect. a Schematic drawing of a PMT. Photons enter through a window on the left side and create free electrons when hitting the photo cathode. These electrons are accelerated due to an external field and consecutively hit different electrodes, where additional electrons are set free. In the end, a single photon entering the PMT creates a measurable signal at the anode. b Schematic drawing of an MCP. In a 2D grid of hollow tubes (channels), to which an electrical field is applied, entering photoelectrons are amplified when they hit the walls of one channel 
Multichannel devices are usually either arrays of photodiodes, which can be fabricated on a single silicon waver, or microchannel plates (Fig. 4b). These provide a 2D grid of amplifying channels that each work similar to a PMT. The total amplification is lower, but unlike PMTs, the resulting information remains spatially resolved after amplification due to the $2 \mathrm{D}$ structure.

It must be noted that in order to operate one of these device in photon-counting mode, additional hardware is necessary to record and save all the acquired pulses with an accurate time stamp.

\subsubsection{Integrating Detectors}

Photon counting devices require very low light levels and expensive read-out electronics. For intense light fluxes, integrating detectors are used. In these, a capacitance is loaded via the photoelectric effect (integration). The charge stored in the capacitance is linearly related to the number of incident photons. It is sampled via an analog-digital converter after the exposure time.

To date, the most common integrating photon detector is the charge-coupled device (CCD), used for example in digital photo and video cameras. CCDs can be manufactured for high sensitivity, even in the NIR range, with up to $95 \%$ quantum efficiency. To obtain maximum sensitivity, they need to be cooled to reduce dark noise. Also, the CCD as a whole has limited dynamics due to the limited well capacities during image exposure.

Another type available nowadays is the CMOS array sensor. These are comprised essentially of a miniaturized photodiode array, including integrators, amplifiers, and readout electronics. CMOS arrays are integrating devices with high dynamics, but not yet as sensitive as CCDs.

Characteristic parameters of integrating detectors are:

- Quantum Efficiency: The probability that a single photon at a given wavelength will interact with the sensor and thus create a signal. For back-illuminated CCDs, this value can be in the order of $95 \%$.

- Full Well Capacity (FWC): Only a limited number of electrons can be stored per pixel. If this number is reached, the detector is saturated. The full well capacity determines the maximum dynamics of the sensor.

- Read Noise (RN): The noise of the analog-to-digital (A/D)-converter. This effectively reduces the sensitivity of the sensor.

- Dark Current (DC): By heat dissipation, electrons are randomly stored in each pixel, thus limiting exposure times and sensitivity. Dark noise is effectively reduced by cooling. Sensitive CCD cameras are usually cooled to below $-50^{\circ} \mathrm{C}$, reducing dark noise to about 0.001 electrons per pixel per second of exposure time.

- Digitizing Accuracy (DA): The A/D converters used can have different resolutions, ranging from 8 bits per pixel in simple cameras to 16 bits per pixel in highly sensitive CCDs. 
The dynamic range (DR) of an array sensor is limited by the last four properties stated above, and can be calculated according to the formula:

$$
D R=\min \left\{\log _{2} \frac{F W C}{D C \cdot E X P+R N}, D A\right\}
$$

The unit of dynamic range, in this case, is bits per pixel. The unit of the full well capacity (FWC) is electrons per pixel, the dark current (DC) is expressed in electrons per pixel per second, the exposure time (EXP) in seconds, and the read noise (RN) in electrons per pixel.

\subsubsection{Intensified and Time-Resolved Imaging}

Due the limited bandwidth and sensitivity of A/D-converters, neither CCD nor CMOS sensors can be read out fast enough to yield a time resolution suitable for resolving photon propagation. However, if the process that is to be observed is repeatable, time-resolved imaging becomes possible by using image amplifiers that can be activated within a few picoseconds, so-called gated imaging. The combination of a light amplifier and a CCD is often called intensified CCD (ICCD).

Light amplification is usually performed using a micro-channel plate (MCP) with a scintillating material at the output which is imaged by the CCD. MCPs can be gated by modulating their operating voltage. The gate widths achievable to date are in the order of $100 \mathrm{ps}$.

Instead of using two separate units for light amplification and detection, both can also be combined on a single integrated circuit. These devices are called electronbombardment (EB) or electron-multiplying (EM) CCDs, depending on the manufacturer. They do not yet achieve the high gain rates of current image intensifiers or ICCDs, nor the short gating times. However, they are much more cost-effective and easier to use.

A mechanical, time-resolved technique involving an integrating detector is the streak camera. It is usually based on a one-line CCD, sometimes also a complete 2D CCD. Incoming photons are swept over the pixels using a deflector, such that every column of the $\mathrm{CCD}$ corresponds to a certain time point after triggering. These devices offer very high temporal resolution below $1 \mathrm{ps}$, even for single shots, but cannot acquire complete 2D images over time.

\section{Microscopic Imaging Techniques}

The following techniques are termed "microscopic" as they offer high resolution (a few microns or less) but only limited depth penetration, so that their application to in vivo settings is limited. Generally, resolution is limited by Abbe's diffraction limit (Hell 2003):

$$
d=\frac{\lambda}{2 \mathrm{NA}}
$$


with $d$ being the shortest distance at which two distinct objects can be separated, and NA is the numerical aperture of the lens used. While the aperture NA can be increased using oil immersion lenses, it is always less than 1 . However, in this section we will also present new developments that overcome the limitations of Abbe's law.

\subsection{Fluorescence Microscopy}

In classical fluorescence microscopy, a single objective is used for illumination and detection at the same time. Excitation light is filtered out using a combination of a dielectric mirror and two (interference) filters (Fig. 5a). In acquired images, fluorescent structures located on the focal plane of the objective appear with high contrast and intensity. The intensity of structures at a distance $r$ from the focal plane decays with $r^{2}$. As fluorochromes throughout the imaged object are excited, out-of-focus signals heavily disturb images, just as in ordinary light microscopy.

Adding temporal resolution to fluorescence microscopy leads to fluorescence lifetime imaging (microscopy) [FLI(M)]. Available as a microscopic as well as a macroscopic technique, lifetime imaging concentrates on the sensitivity of a fluorochrome (and of its lifetime) to the environment, e.g., $\mathrm{pH}$ value. As a source, either pulsed or modulated light is used. For pulsed light, the observed fluorescence decay is multi-exponential, with different exponents for the different lifetimes in the sample. For modulated light, phase shifts are observed.

a

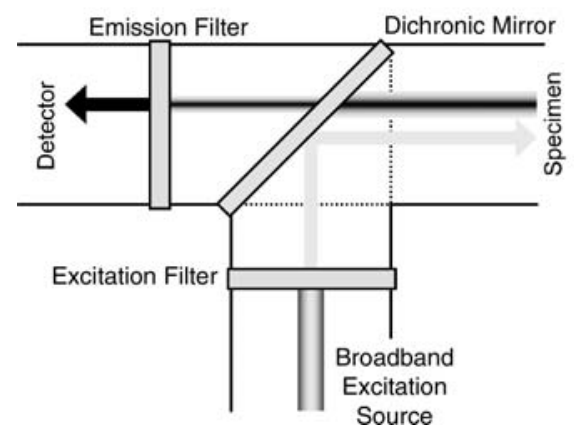

b

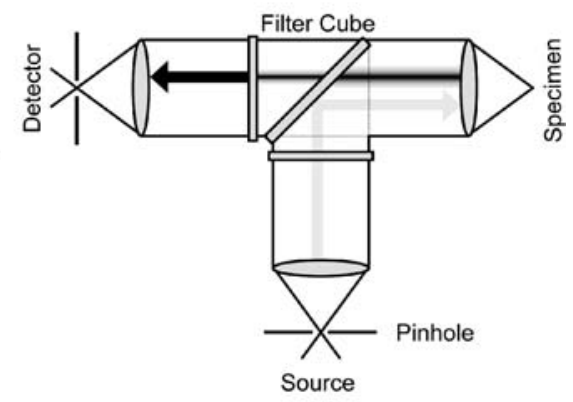

Fig. 5 a, b Principle of fluorescence and confocal microscope. a Schematic drawing of a filter cube as employed in fluorescence microscopes. Light from a source is filtered and reflected onto the sample by a dichroic mirror. Reflected fluorescence light is transmitted through the mirror and filtered, then guided onto the detector where a full image can be recorded. b In confocal scanning microscopy, the image of a point source is produced inside the sample. Only fluorescence light emitted from this focal spot is detected by the detector due to the presence of an additional pinhole aperture 


\subsection{Confocal Microscopy}

To overcome the limitations of fluorescence microscopy, i.e., to limit detected signals to the focal plane (or to a focal spot) thus allowing full 3D scanning through the specimen, confocal microscopy was developed. Here, the specimen is not evenly illuminated. Instead, excitation light is focused onto a single point; detection is performed using basically the same optics as in standard fluorescence microscopy, including a pinhole aperture cutting away light originating from outside the focal spot (Fig. 5b). This single-point-illumination, single-point-detection technique allows scanning of the focal spot through the whole specimen, as long as there is only little scattering to disturb the appearance of the focus. Of course, necessary light intensities are high and scan times are long, so that photobleaching can become an issue when using fluorescent probes.

In order to further improve resolution, two-photon microscopy can be used. When two photons of approximately double the single-photon excitation wavelength interact with a fluorescent molecule within a very short time span, they can excite the molecule. The probability of two photons arriving simultaneously depends nonlinearly on light intensity. A laser beam of low photon flux is focused such that only in the focal spot the necessary photon density is reached to excite two-photon fluorescence (Helmchen and Denk, 2005) such that detected fluorescence signals originate exclusively from this small region. Three-dimensional images are obtained by scanning the focal spot over and into the specimen. In two-photon microscopy, light intensities are even stronger than in confocal microscopy, further increasing photobleaching and tissue damage issues.

\section{$4.34 \pi$ Microscopy}

The resolution in confocal microscopy is anisotropic: while it is about $250 \mathrm{~nm}$ in the focal plane, in axial direction it decreases to roughly half the resolution, about $500 \mathrm{~nm}$. If, however, not one laser beam is focused, but if the beam is split and then focused from two sides, the focal spot will show an interference pattern (standing wave) with a strong central spot and smaller side lobes (Fig. 6). The central spot is much smaller than the original focal spot, thus increasing resolution if the side lobe signal is eliminated using deconvolution techniques. This technique can yield an isotropic resolution of about $100 \mathrm{~nm}$ (Hell 2003).

\subsection{Stimulated Emission Depletion (STED)}

An even higher resolution can be achieved using fluorescence depletion. Immediately following a very short excitation light pulse, another high-intensity light pulse at the emission wavelength is sent towards the sample. The time span between first 

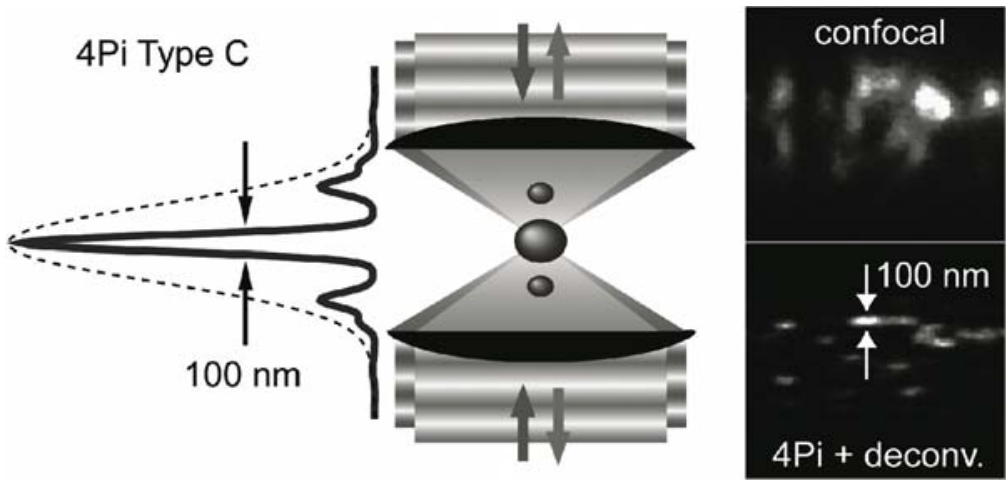

Fig. 6 Working principle of a $4 \pi$ microscope and exemplary results. Left: Two lenses are used for focal spot creation from two opposing sides. As coherent light is used, a standing wave pattern evolves, having a small central maximum and several side lobes. The central lobe is significantly smaller than the size of the original focal spot in confocal microscopy. Side lobes have to be removed using deconvolution techniques. Right: Exemplary results, comparing cellular structures obtained with confocal and $4 \pi$ microscopy, demonstrating the improved resolution. (Images kindly provided by Marion Lang, German Cancer Research Center, Heidelberg)

and second pulse needs to be shorter than the fluorescence lifetime. The second pulse is required to be sufficiently intense to force depletion of excited fluorescent molecules by stimulated emission. By use of a phase shifting plate, the shape of the focal region of the second pulse can be changed so that a very small central region is spared from depletion. Fluorescent signals originating from this region can still be detected after the depletion pulse. The size of this region can be less than $100 \mathrm{~nm}$; a resolution of up to $50 \mathrm{~nm}$ seems realistic (Hell 2003). A schematic drawing of the instrument and the size of the source spot, which determines resolution, is depicted in Fig. 7.

\subsection{Other Microscopic Techniques}

Beside the techniques described in the paragraphs above, a couple of further microscopic imaging modalities are worth mentioning that try to overcome the diffraction limit. While the $4 \pi$ or STED microscope use interference effects to reduce the size of the focal spot, structured illumination microscopy is a non-scanning, wide-field technique. Different excitation light patterns are used to excite fluorochromes; postprocessing of the resulting images can yield highly resolved images. However, the spatial frequencies that can be contained in the excitation pattern are also bandlimited due to Abbe's law. If again, as in STED or two-photon microscopy, nonlinear effects exist in the excitation process, e.g., saturation effects, higher spatial frequencies will be contained in the emission and can be extracted in the postprocessing (Gustafsson 2005). 


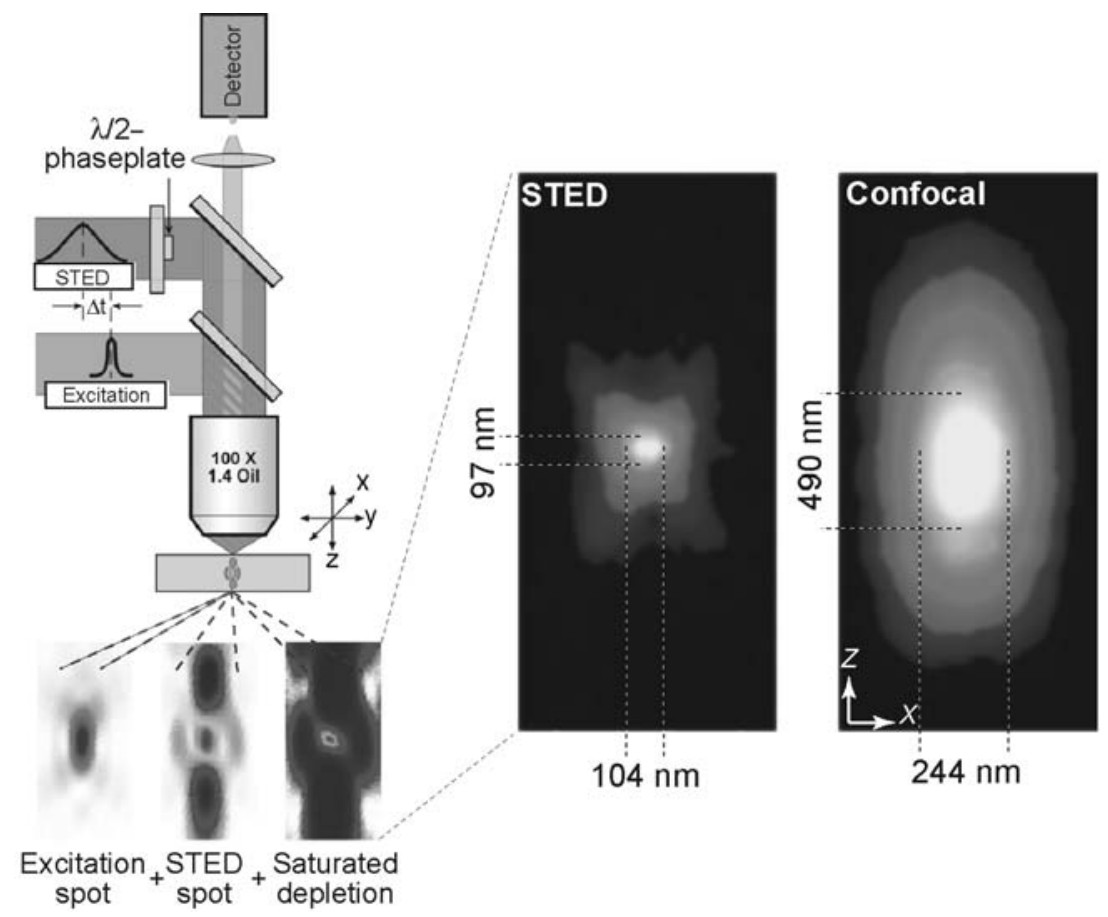

Fig. 7 Left: Schematic drawing of a STED microscope. The microscope is operated with two pulsed light sources, gated shortly after one another. The firs pulse excites the sample in the focal spot. The second pulse is directed through a phase plate to change appearance of the focal spot and depletes the excited fluorochromes in a region surrounding the focal spot. Only the remaining region can then spontaneously emit fluorescence photons. Right: Size and shape of the fluorescent spot in STED and confocal microscopy, showing significant resolution improvement. (Adapted from Hell 2003)

Microaxial tomography, as another candidate, extends confocal microscopy not to enhance resolution in the focal plane, but only axially, to get a more isotropic resolution. The imaged specimen, e.g., a cell, is fixed on the outside of a glass tube and rotated within the field of view of the confocal microscope. Thus, several sets of confocal 3D data are acquired, all having their own highly resolved focal plane; as those focal planes are not parallel to each other, tomographic reconstruction methods can be employed to reduce the size of the focal spot to the intersection of all focal spots. If images are acquired from $360^{\circ}$, the focal spot reduces to a sphere, yielding isotropic resolution (Heintzmann and Cremer 2002). A last technique to be mentioned is optoacoustic microscopy (Xu and Wang 2006). It is based on the photoacoustic effect, mentioned in more detail below. 


\section{Whole-Animal Imaging Techniques}

\subsection{Bioluminescence and Fluorescence Imaging}

Acquiring bioluminescence signals in whole animals is rather trivial: all one needs, apart from suitable cell lines, is a light-tight chamber and a very sensitive CCD camera (Fig. 8). As no autofluorescence background or filter leakage from excitation light disturbs the actual signal, images are of rather good quality. It is possible to track very few cells even relatively deep inside the tissue (Massoud and Gambhir 2003).

Fluorescence reflectance imaging (FRI) requires additionally light sources to excite fluorochromes, and filters to eliminate the excitation light. Excitation and detection are performed on the same side of the imaged object, in reflectance geometry. As excitation light intensity as well as the sensitivity for fluorescence light decay exponentially with depth in tissue, this imaging modality is highly surfaceweighted (Weissleder and Ntziachristos 2003). Filter leakage is a major problem, as a significant amount of excitation light is already reflected before entering the uppermost skin layer.

Yet another method to display fluorescent inclusions in tissue is to use transillumination instead of reflectance (Zacharakis et al. 2006). Here, excitation and detection are performed from opposite sides. Images are less surface-weighted as excitation light intensity decreases exponentially towards the detector while fluorescence sensitivity increases. Results can be further enhanced by "normalizing" acquired fluorescence images with images showing only excitation light. Thus, heterogeneities due to high absorption in tissue are reduced.

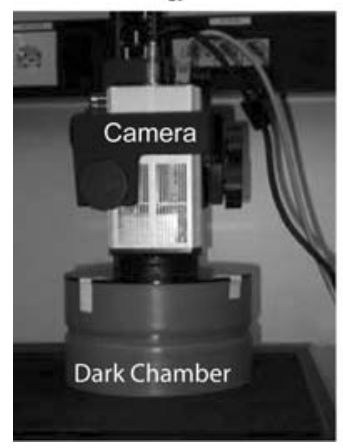

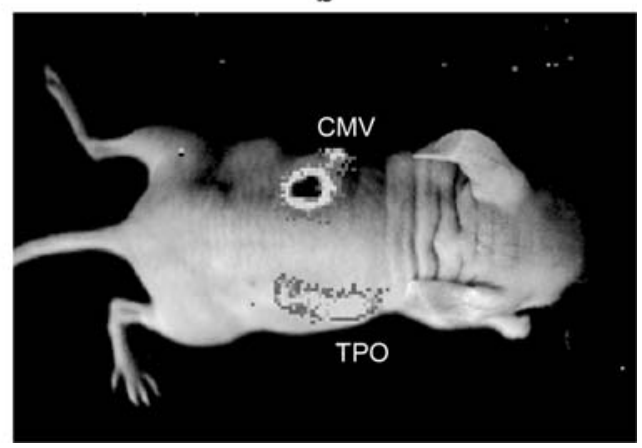

Fig. 8 a, b Bioluminescence imaging (BLI) of whole animals. a The necessary setup for BLI consists only of a dark chamber for animal placement and a sensitive CCD-camera; this setup is nowadays commercially available from a number of companies. b Sample result of bioluminescent tumors in a nude mouse. Modified Morris hepatoma cells were subcutaneously implanted as tumors on the left and right dorsal side of immunodeficient mice. Cells were modified to express firefly luciferase, tagged to different gene promoters (CMV and TPO, respectively) 


\subsection{Bioluminescence and Fluorescence Tomography}

Tomographic imaging in optics requires a mathematical means of contributing a photon density distribution measured on the outer boundaries of the imaged object to absorbers, scatterers, or source inside the object; this is termed the inverse problem. Usually, the inverse problem is given via the direct problem: for a given propagation model, one tries to estimate a set of model parameters that give results fitting the actual measurements. The most common model used is the diffusion equation (Gibson et al. 2005):

$$
\left[\nabla \frac{1}{3(1-g) \mu_{s}(\mathbf{r})} \nabla+\mu_{a}(\mathbf{r})\right] \Phi(\mathbf{r})=-q(\mathbf{r})
$$

In (3), $\mathbf{r}$ is the spatial coordinate, $\Phi$ is the photon density distribution, $q$ is the photon source distribution, and $\mu_{s}$ and $\mu_{a}$ are scattering and absorption coefficients, respectively. The factor $(1-g)$, ranging between 0 and 2 , accounts for the possibly anisotropic character of scattering. For purely isotropic scattering it is 1, for pure back-scattering it is 0, and for pure forward scattering, it is 2. Equation (3) models both, photon propagation from the excitation source as well as emitted photons. In the first case, $q$ describes the light input and the model results in a photon distribution $\Phi_{\mathrm{x}}$ that can excite fluorochromes. To model fluorescence, $q$ is then replaced by $\Phi_{\mathrm{X}}$ times the quantum efficiency and absorption (concentration times extinction) of the fluorochrome, i.e., $q_{m}(\mathbf{r})=\gamma \varepsilon c(\mathbf{r}) \Phi_{x}(\mathbf{r})$.

Reconstruction is a process to estimate either $\mu_{s}$ and $\mu_{a}$, or alternatively to calculate the concentration $c$. Usually, for fluorescence tomography $\mu_{s}$ and $\mu_{a}$ are assumed to be known and constant which simplifies the model dramatically. Nevertheless, reconstruction is mathematically challenging and time consuming. On the other hand, experimental acquisition of diffuse projections is a rather simple task, basically employing a laser diode source and a CCD coupled to the object either by an objective lens or by a number of fiber detectors mounted on some kind of gantry to enable transillumination of the object or animal from different directions, as shown in Fig. 9.

An important property of optical tomographic systems is whether light coupling is performed using fibers in contact with the imaged object, or whether contact-free detection via an objective lens is implemented (noncontact imaging; Schulz et al. 2004). While in fiber-based designs, complex shaped objects have to be embedded in some kind of optically matching fluid as the fiber ends usually cannot be positioned arbitrarily, this is unnecessary for non-contact designs. The embedding itself has another advantage as it simplifies the boundary conditions for the PDE and thus simplifies the reconstruction while attenuating the signal and making the mapping to anatomy more difficult in the end (when animals are imaged that have to be mounted floating in the matching fluids). Lens-coupled detection in turn offers much more channels, as every pixel of the CCD can be used, and enables imaging without matching fluid if and only if the object geometry is known (or can be acquired using a 3D scanning system) and thus appropriate boundary conditions can be applied. 


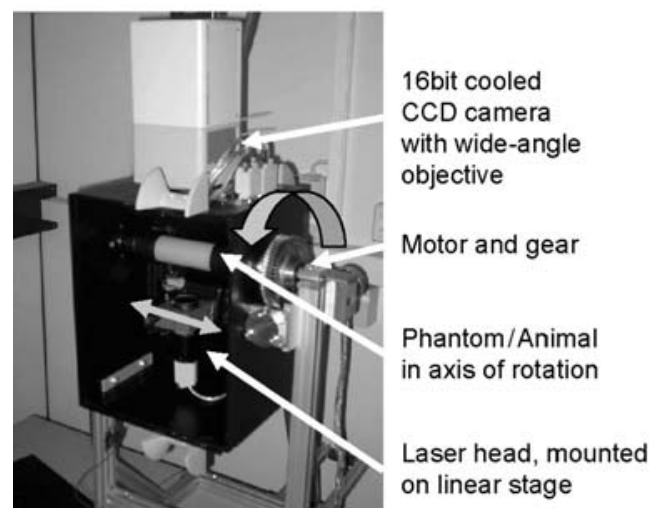

b

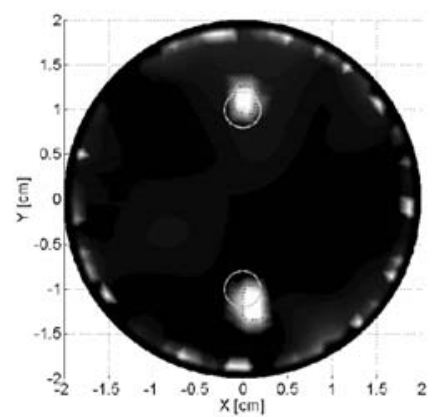

Fig. 9 a A typical non-contact optical tomography system, consisting of a sensitive CCD camera and a laser source, rotating around the imaged specimen. $\mathbf{b}$ Central transversal slice of a rat-sized diffuse phantom containing two fluorescent inclusions. Actual inclusion positions are denoted by circles. (Image courtesy of the authors)

For optical tomographic purposes, there exist also an abundance of techniques that employ time-resolved information for the location and quantization of fluorescence in vivo. The interested reader is referred to the review by Dunsby and French (2003).

In fluorescence tomography, usually several different source positions are chosen. For each position the fluorescent molecules located in the tissue are excited differently due to the different light distributions from different source positions. This change in excitation and thus emission pattern means additional information and in fact makes the whole problem of reconstructing concentrations tractable at all.

In bioluminescence tomography there is no excitation source. Therefore, one cannot acquire several different images from bioluminescence and then reconstruct based on the observed differences. Instead, what researchers try to perform is spectral imaging: light attenuation depends on wavelength. If the emission spectrum is known, the deviations of the observed light emissions from this original spectrum can be used to estimate the depth of the bioluminescent source in tissue (e.g., Dehghani et al. 2006). These techniques, however, are still under development; in vivo results are not yet available.

\subsection{Optoacoustic Tomography}

Another emerging imaging technique is optoacoustic tomography, which uses shortly pulsed laser sources for excitation but ultrasound detectors for detection ( $\mathrm{Xu}$ and Wang 2006; Ntziachristos et al. 2005). The absorption of light by tissue or fluorochromes leads to local heating, which in turn leads to an expansion depending 
on the amount of energy absorbed. This expansion will create a pressure (= sound) wave with a frequency in the ultrasound region.

This technique is advantageous over classical fluorescence tomography as ultrasonic reconstruction can be performed more easily than optical reconstruction, albeit it is more complex than standard ultrasound imaging as it is based on the diffusion equation as well, not on mere echo times. Optoacoustic tomography is capable of showing anatomic details, however, this might decrease its sensitivity for specific probes as probe signal and tissue signal have to be dissolved. For details on the technique, please refer to Wang (2003-2004).

\section{Summary}

Optical imaging offers unique possibilities for in vitro and in vivo imaging applications, especially in the context of molecular imaging. Understanding the fundamentals of optical imaging and grasping the pros and cons of available imaging techniques is a must for researchers interested in the field. This chapter reviewed the basic concepts of optical imaging instrumentation as well as state-of-the-art imaging techniques. For more detailed discussions of the subject, the reader is kindly referred to the articles below.

\section{References}

Dehghani $\mathrm{H}$ et al (2006) Spectrally resolved bioluminescence optical tomography. Opt Lett 31:365-367

Dunsby C, French PMW (2003) Techniques for depth-resolved imaging through turbid media including coherence-gated imaging. J Phys D: Appl Phys 36:R207-R227

Gibson AP, Hebden JC, Arridge SR (2005) Recent advances in diffuse optical imaging. Phys Med Biol 50:R1-R43

Gustafsson MGL (2005) Nonlinear structured-illumination microscopy: wide-field fluorescence imaging with theoretically unlimited resolution. Proc Natl Acad Sci USA 102:13081-13086

Heintzmann R, Cremer C (2002) Axial tomographic confocal fluorescence microscopy. J Microsc 206:7-23

Hell SW (2003) Toward fluorescence nanoscopy. Nat Biotechnol 21:1347-1355

Helmchen F, Denk W (2005) Deep tissue two-photon microscopy. Nat Methods 2:932-940

Hielscher AH (2005) Optical tomographic imaging of small animals. Curr Opin Biotechnol 16:79-88

Hoffman RM (2005) The multiple uses of fluorescent proteins to visualize cancer in vivo. Nat Rev Cancer 5:796-806

Kraayenhof R, Visser AJWG, Gerritsen HC (2002) Fluorescence spectroscopy, imaging and probes: new tools in chemical, physical and life sciences. Springer, Berlin Heidelberg New York

Lakowicz JR (2006) Principles of fluorescence spectroscopy, 3rd edn. Springer, New York

Massoud TF, Gambhir SS (2003) Molecular imaging in living subjects: seeing fundamental biological processes in a new light. Genes Dev 17:545-580

Mobley J, Vo-Dinh T (2003) Optical Properties of Tissue. In: Vo-Dinh T (2003) Biomedical photonics handbook. CRC Press, Boca Raton, pp 2/1-2/75 
Mycek M-A, Pogue BW (2003) Handbook of biomedical fluorescence. Marcel Dekker, New York Ntziachristos V et al (2005) Looking and listening to light: the evolution of whole-body photonic imaging. Nat Biotechnol 23:313-320

Redmond RW (2003) Introduction to fluorescence and photophysics. In: Mycek M-A, Pogue BW (eds) Handbook of biomedical fluorescence. Marcel Dekker, New York, pp 1-28

Schulz RB, Ripoll J, Ntziachristos V (2004) Experimental fluorescence tomography of tissues with non-contact measurements. IEEE Trans Med Imaging 23:492-500

Shaner NC, Steinbach PA, Tsien RY (2005) A guide to choosing fluorescent proteins. Nat Methods 2:905-909

Vo-Dinh T (2003a) Biomedical photonics handbook. CRC Press, Boca Raton

Vo-Dinh T (2003b) Basic instrumentation in photonics. In: Vo-Dinh T (2003) Biomedical photonics handbook, CRC Press, Boca Raton, pp 6/1-6/30

Wang LV (2003-2004) Ultrasound-mediated biophotonic imaging: a review of acousto-optical tomography and photo-acoustic tomography. Dis Markers 19:123-138

Weissleder R, Ntziachristos V (2003) Shedding light onto live molecular targets. Nat Med 9:123-128

Xu M, Wang LV (2006) Photoacoustic imaging in biomedicine. Rev Sci Instrum 77:41-101

Zacharakis $\mathrm{G}$ et al (2006) Normalized transillumination of fluorescent proteins in small animals. Mol Imaging 5:153-159 